\title{
Axial ion charge state distribution in the vacuum arc plasma jet
}

\author{
M. Keidar ${ }^{\mathrm{a})}$ \\ Department of Aerospace Engineering, University of Michigan, Ann Arbor, Michigan 48109 \\ I. G. Brown \\ Lawrence Berkeley National Laboratory, University of California, Berkeley, California 94720 \\ I. I. Beilis \\ Electrical Discharge and Plasma Laboratory, Tel Aviv University, Tel Aviv 69978, Israel
}

(Presented on 6 September 1999)

\begin{abstract}
We report on our experimental studies of the ion charge state distribution (CSD) of vacuum arc plasmas using a time-of-flight diagnostic method. The dependence of the CSD on the axial distance from the plasma source region was measured for a titanium vacuum arc. It was found that the axial CSD profile is nonuniform. Generally, the mean charge state increases approximately linearly with axial distance from about 1.7 at $12 \mathrm{~cm}$ up to 1.9 at $25 \mathrm{~cm}$ from the plasma source. A model for ion transport in the free boundary plasma jet is proposed which is based on the existence of an electric field in the quasineutral plasma. This model qualitatively explains the experimental results. (C) 2000 American Institute of Physics. [S0034-6748(00)58302-6]
\end{abstract}

\section{INTRODUCTION}

The generation of multiply charged ion beams is an active research area with many applications. Broad beams of multiply charged metal ions can be produced by a vacuum arc plasma discharge. ${ }^{1}$ The properties and characteristics of the vacuum-arc-generated plasma have been investigated for many decades. ${ }^{2}$ One of the advantages of the vacuum arc is the formation of a relatively high fraction of multiply charged ions. The charge state distribution (CSD) of ions produced by vacuum arc plasma jets contains species with $Q=1$ up to 5 , with a mean charge state $\langle Q\rangle=1-3$ under normal conditions depending on the cathode material used. ${ }^{3}$ Application of a strong external magnetic field and operation with high arc current have been found to be effective ways to increase the ion charge state distribution. ${ }^{4,5}$

The vacuum arc plasma is generated at cathode spots. The plasma expands from the cathode spots into the vacuum and, in an ion source embodiment, streams toward the ion extractor system. The plasma expansion has been studied theoretically previously in terms of a free-boundary plasma jet. ${ }^{6}$ An ambipolar electric field exists in the expanding plasma and affects the highly charged ion species more than the lower charge states. It has been predicted that ion species with different charge states may be separated in the free boundary plasma jet so that the radial CSD becomes nonuniform. ${ }^{6}$ It has also been shown that the effect of the spatial separation of different charged species depends on axial distance from the plasma generation region.

Some experimental data on the CSD in the stationary vacuum arc plasma jet have previously been obtained by mass spectrometry. ${ }^{7}$ It was found that along the centerline the mean charge state decreases significantly $30 \mathrm{~cm}$ from the cathode plane. Unfortunately, the off-centerline axial varia-

${ }^{\text {a)} E l e c t r o n i c ~ m a i l: ~ k e i d a r @ e n g i n . u m i c h . e d u ~}$ tion of the CSD was not presented. Here we report on the results of some experimental investigations of the axial charge state distribution using a time-of-flight diagnostic, and compare these results with theoretical predictions.

\section{EXPERIMENTAL SETUP AND RESULTS}

Our experiments were carried out using a vacuum arc ion source of a kind that has been described in detail elsewhere ${ }^{8}$ here we present only a brief description of the particular embodiment employed in the present work. The vacuum arc minigun consists of a cathode, an anode, and a trigger. The cathode spots burn on the front surface of a cylindrical titanium cathode ( $6.25 \mathrm{~mm}$ diameter), the annular anode has an inner diameter of about $2 \mathrm{~cm}$, and the cathodeanode distance is about $1 \mathrm{~cm}$. For the work described here the arc current was pulsed, of $100 \mathrm{~A}$ amplitude and $250 \mu \mathrm{s}$ duration. The ion beam extractor voltage was $25 \mathrm{kV}$, and the distance between plasma source and extractor could be varied from 10 to $25 \mathrm{~cm}$.

The CSD was investigated by a time-of-flight (TOF) charge-to-mass spectrometer system that is described elsewhere. ${ }^{9}$ The beam passes through an annular electrostatic beam deflector, with a central plate to block the direct line of sight between plasma the gun cathode and the TOF Faraday cup. The accelerated ions drift through a distance $(\sim 1 \mathrm{~m})$ that is sufficient to allow the different charge-to-mass ion species to be spatially separated. The ions are monitored by a Faraday cup at the far end of the drift region (as shown in Fig. 1) that serves as a current monitor. The charge state distribution is determined by the relative pulse heights of the TOF spectrum.

Thus, for this work, the ion beam formation system together with the time-of-flight charge state diagnostic system serve as a means for characterizing the charge state distribution of the vacuum arc plasma as a function of axial position within the vacuum arc plasma jet. The axial position moni- 


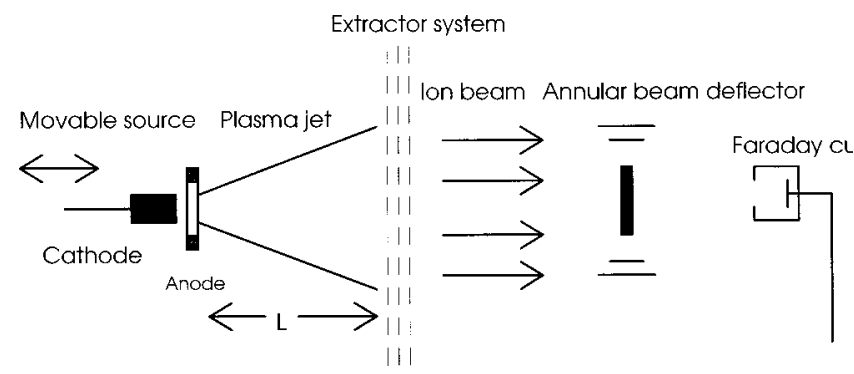

FIG. 1. Schematic of the vacuum arc plasma source and TOF diagnostics.

tored was varied simply by varying the distance from the plasma gun to the extractor. Some variation of the CSD from shot to shot was observed, especially at small distances from the plasma source. All results presented here were averaged over 256 shots for each measurement.

A plot showing the typical variation of the individual charge state fractions with axial distance is shown in Fig. 2. At the closest distance to the plasma source, high charge states were measured. In general the singly charged ion flux decreases with axial distance while the doubly and triply charged ion fluxes increase. This effect can be seen explicitly in Fig. 3, where the axial variation of mean charge state is plotted. The mean charge state increases approximately linearly with axial distance from about 1.7 at $12 \mathrm{~cm}$ up to 1.9 at $25 \mathrm{~cm}$ from the plasma source. The high mean charge states measured at the closest distance to the cathode plane may be due to the influence of the high voltage extractor system on the plasma jet. In fact, at this distance large fluctuations in the CSD were observed.

\section{MODEL AND COMPARISON WITH EXPERIMENT}

We now briefly describe a model for the plasma jet expansion, with which we can simulate the above experiment. A detailed description of the model can be found in Ref. 6 . The model deals with the plasma jet originating from the cathode. According to the vacuum arc cathode jet model the plasma has a density of about $10^{20} \mathrm{~m}^{-3}$ in the interelectrode gap at a distance of a few $\mathrm{cm}$ from the cathode plane. ${ }^{10}$ For such a density range the influence of inelastic collisions (ionization, recombination, etc.) on the plasma density can be

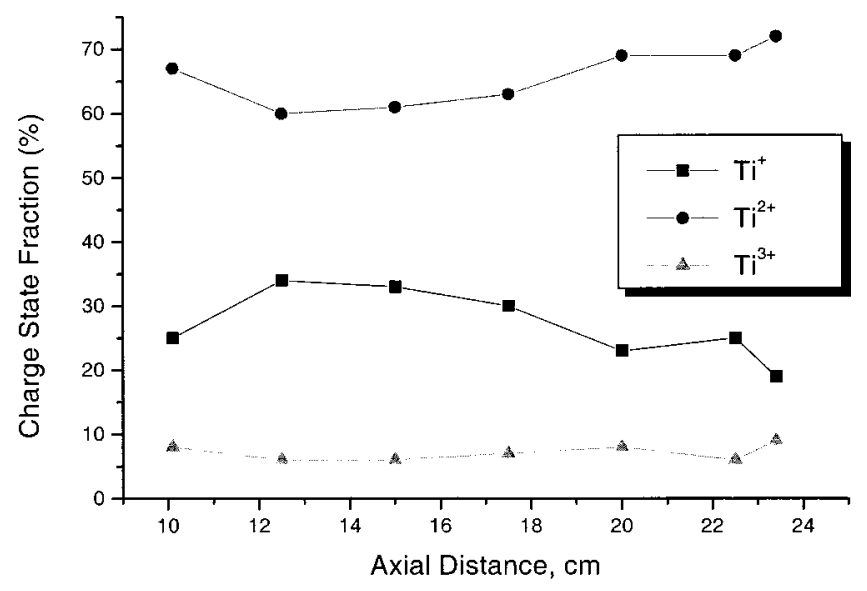

FIG. 2. Charge state fractions as a function of axial distance from the plasma source.

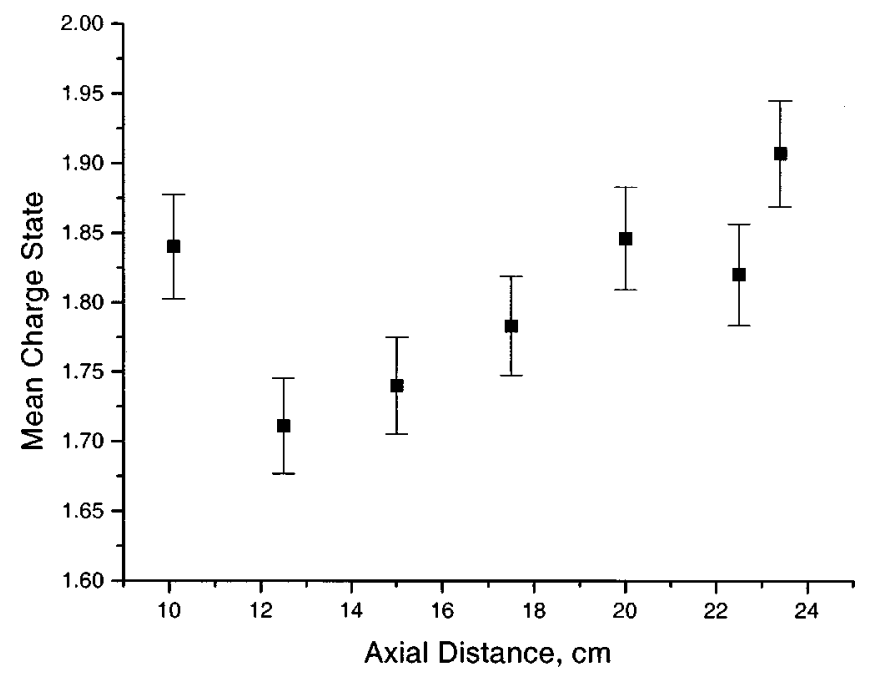

FIG. 3. Mean charge state as a function of distance from the plasma source.

neglected. Experimental and theoretical investigations show that the vacuum arc plasma is almost fully ionized. ${ }^{2,11}$ The plasma jet has a free radial boundary whose position will be determined as part of a self-consistent solution. ${ }^{11}$ We will employ the following assumptions and conditions: (i) the vacuum arc plasma is fully ionized; (ii) during the expansion, the temperature of each species remains constant; (iii) the mean free path for elastic electron-ion collisions is much smaller than the characteristic plasma jet radius; (iv) the ions and electrons are ideal gases with partial pressures $P_{\alpha}$ $=k T_{\alpha} n_{\alpha}$, where $\alpha=e$ and, $i, k$ is the Boltzmann constant, and $T_{e}$ and $T_{i}$ are the electron and ion temperatures, respectively; (v) the plasma does not carry electrical current, $J$ $=0$; (vi) no magnetic field is applied. The steady state behavior of such a quasineutral, multicomponent plasma is described by the following set of equations (subscript $j$ corresponds to the different ion charge-state species. $j=1,2,3,4)$ :

$$
\begin{aligned}
& m_{j} n_{j}\left(\mathrm{v}_{j} \nabla\right) \mathrm{v}_{j}=Q_{j} e n_{j} E-\nabla P_{j}-m_{e} n_{j} \nu_{j e}\left(\mathrm{v}_{j}-\mathrm{v}_{e}\right) \\
& 0=-e n_{e} E-\nabla P_{e}+m_{e} n_{e} \sum \nu_{e j}\left(\mathrm{v}_{i}-\mathrm{v}_{e}\right) \\
& \nabla\left(n_{e} \mathrm{v}_{e}\right)=0 \\
& \nabla\left(n_{j} \mathrm{v}_{j}\right)=0
\end{aligned}
$$

where $\mathbf{v}_{j}$ and $\mathbf{v}_{e}$ are the ion and electron velocities, respectively, $\nu_{e j}$ is the collision frequency for electrons with ions of charge $Q_{j}$, and $n_{j}$ is the density. From Eqs. (1) and (2) using the quasineutrality condition $n_{e}=\Sigma Q_{j} n_{j}$ and $J$ $=e n_{e} \mathbf{v}_{e}-\Sigma Q_{j} e n_{j} \mathbf{v}_{j}=0$ we have the equation for electric field in the quasineutral plasma region:

$$
E=-\frac{k T_{e}}{e} \nabla \ln \left(\sum Q_{j} n_{j}\right)-n_{e} m_{e} \sum \nu_{e j}\left(\mathrm{v}_{j}-\mathrm{v}_{e}\right) .
$$

The numerical analysis used here is similar to that developed previously.,11 The system of Eqs. (1)-(4) was solved by a finite difference method. The equations were approximated by a two-layer, implicit six point scheme. ${ }^{12}$ The problem was analyzed using dimensionless variables, where all velocities are normalized to the sonic velocity $c_{s}$ 


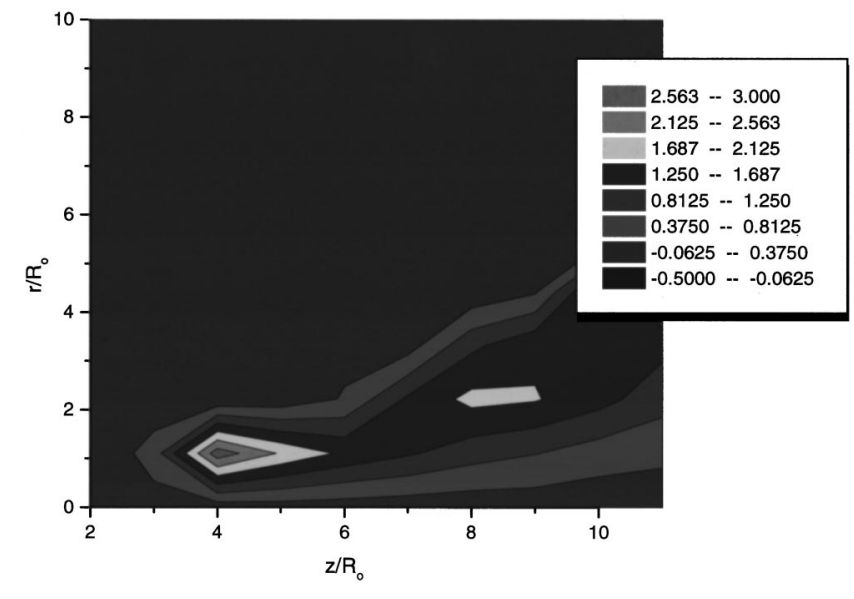

FIG. 4. Normalized radial component of electric field.

$=\left[\left(k T_{e}+k T_{i}\right) / m_{i}\right]^{0.5}$, the density to its value at the starting plane, $n_{0}$, and all coordinates to $R_{0}$, where $R_{0}$ is the plasma jet radius at the starting plane.

The calculated radial component of the electric field in the plasma expansion region is shown in Fig. 4. The electric field generally decreases in the axial direction. One can see that the normalized electric field is about 1 . For the case considered, $T_{e}=2 \mathrm{eV}$ and $R_{0}=0.015 \mathrm{~m}$, the radial component of electric field lies in the range of 100-400 V/m.

The axial distribution of the mean ion charge state along the centerline and in the plasma jet boundary region is shown in Fig. 5. It can be seen that mean charge state decreases with axial distance from the starting plane along the centerline, and increases in the plasma jet boundary region. For comparison we have plotted our experimental data for the mean charge state variation (triangles) and also the charge states measured by Khoroshich et al. ${ }^{7}$ along the centerline (squares). One can see that the predictions of our model of the charge state behavior in the near boundary region agree

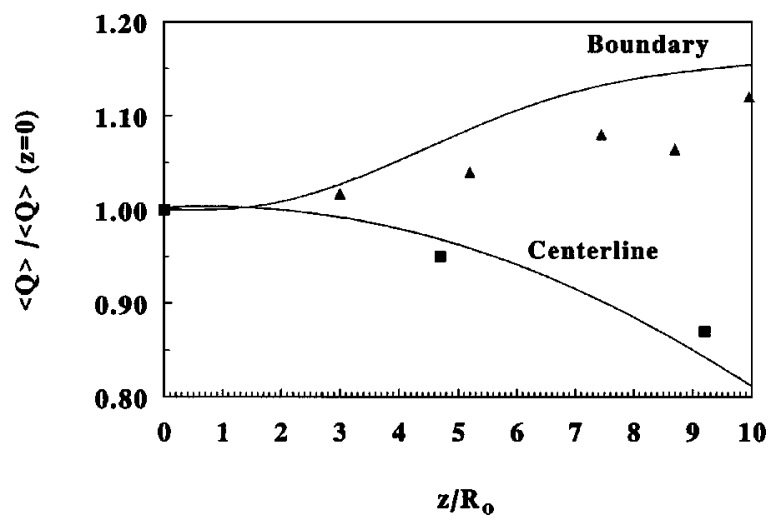

FIG. 5. Mean charge state axial distribution. The solid line refers to the prediction of the model. The experimental data: present work (triangles); Khoroshikh et al. (Ref. 7) (squares). with the measurements carried out by the time-of-flight technique (see Sec. II), while the centerline charge state variation agrees with mass-spectrometer measurements. ${ }^{7}$ We note that our TOF measurements are probably weighted toward the boundary region of the plasma jet because the TOF gate employed is annular and not on axis; however, this effect is reduced to the extent that the extracted ion beam suffers "radial mixing"' via beam divergence effects.

\section{DISCUSSION}

It was found that the electric field in the plasma expansion region may lead to separation of ion charge state species. However, this is a relatively small effect, since in the free quasineutral plasma jet there is a small electric field of magnitude about $\sim 100 \mathrm{~V} / \mathrm{m}$. This corresponds to a potential drop across the plasma jet of only about $10 \mathrm{~V}$. In the vacuum arc plasma jet ions have a directed energy of about $50 \mathrm{eV},{ }^{13}$ which is larger than the potential drop within the plasma jet. Thus one can expect only a small influence of the electric field on the ion dynamics. However, the effect of ion species separation has some general importance. For instance, the presence of a strong electric field could lead to a much stronger effect. Such an electric field could be generated within the quasineutral plasma by application of an axial magnetic field in the interelectrode region. This electric field has the opposite direction to that considered in the present work. Thus one would expect high-charge-state ions to be pushed toward the axis. This could explain the observed increase in mean ion charge state with axial magnetic field strength. ${ }^{3-5}$

\section{ACKNOWLEDGMENTS}

One of the authors (M.K.) gratefully acknowledges the financial support of the Fulbright and Welch fellowship programs. This work was supported by the U.S. Department of Energy under Contract No. DE-AC03-76SF00098.

${ }^{1}$ I. G. Brown, Rev. Sci. Instrum. 65, 3061 (1994).

${ }^{2}$ Handbook of Vacuum Arc Science and Technology, edited by R. L. Boxman, D. M. Sanders, and P. J. Martin (Noyes, Park Ridge, 1995).

${ }^{3}$ I. G. Brown and X. Godechot, IEEE Trans. Plasma Sci. 19, 713 (1991).

${ }^{4}$ E. M. Oks, A. Anders, I. G. Brown, M. R. Dickinson, and R. A. MacGill, IEEE Trans. Plasma Sci. 24, 1174 (1996).

${ }^{5}$ E. M. Oks, I. G. Brown, M. R. Dickinson, R. A. MacGill, H. Emig, P. Spadtke, and B. H. Wolf, Appl. Phys. Lett. 67, 200 (1995).

${ }^{6}$ M. Keidar, I. I. Beilis, and I. G. Brown, J. Appl. Phys. 84, 5956 (1998).

${ }^{7}$ V. M. Khoroshikh, I. I. Aksenov, and I. I. Konovalov, Sov. Phys. Tech. Phys. 33, 723 (1998).

${ }^{8}$ I. G. Brown, in The Physics and Technology of Ion Sources, edited by I. G. Brown (Wiley, New York, 1989).

${ }^{9}$ I. G. Brown, J. E. Galvin, R. A. MacGill, and R. T. Wright, Rev. Sci. Instrum. 58, 1589 (1987).

${ }^{10}$ I. I. Beilis and M. Zektzer, High Temp. 29, 501 (1991).

${ }^{11}$ M. Keidar, I. Beilis, R. L. Boxman, and S. Goldsmith, J. Phys. D: Appl. Phys. 29, 1973 (1996).

${ }^{12}$ V. M. Paskonov, in Numerical Methods in Gas Dynamics, edited by G. S. Roslyakov and L. A. Chudov (Israel Program for Scientific Translation, Jerusalem, 1966).

${ }^{13}$ J. Kutzner and H. C. Miller, J. Phys. D: Appl. Phys. 25, 686 (1992). 